\title{
Synthesis of New Donor-functionalized Alcohols as Organic Ligands for Metal Precursors of Metal Organic Chemical Vapor Deposition
}

\author{
Minchan Kim, ${ }^{\dagger}$ Hong Suk Jang, Taek-Mo Chung, ${ }^{*}$ Chang Gyoun Kim, ${ }^{*}$ Yunsoo Kim, and Bong Rae Cho ${ }^{\dagger}$ \\ Thin Film Materials Laboratory, Advanced Materials Division, Korea Research Institute of Chemical Technology, \\ Yuseong, P.O. Box 107, Daejeon 305-600, Korea. "E-mail: tmchung@krict.re.kr; cgkim@krict.re.kr \\ †Department of Chemistry, Korea University, Anam-dong, Seoul 136-701, Korea \\ Received January 28, 2005
}

Key Words : MOCVD, Precursor, Nucleophilic additions, Ring opening, Complexes

Recently, the development and use of single precursors for metal organic chemical vapor deposition (MOCVD) has been extensively focused on application to electronic devices. ${ }^{1-6}$ However, systematic design and synthesis of organic ligands appropriate for preparation of metal complexes with relevant chemical and physical properties, such as stability, volatility, and resistance to air and moisture have not received much attention. Generalization to prepare organic ligands for MOCVD precursors is especially important because the large scale synthesis of the metal complexes exclusively depends on the potential usefulness of the ligands. ${ }^{7}$

It is desirable for metal complexes to be in monomeric forms because mononuclear complexes generally show high vapor pressures and low sublimation or boiling temperatures. To do so, the coordinated atom of ligands should be crowded in its own environment with some substituents by keeping the coordinated atoms from bonding to another metal through bridging configuration. Therefore, pendant groups should be introduced into organic ligands.

Some research results have been reported on the synthesis and application of organic ligands containing pendant groups. Herrmann reported his pioneering works on the synthesis of donor-functionalized alcohols and their application as bidentate ligands for the preparation of metal complexes. ${ }^{8-10}$ A variety of donor-functionalized alcohols which contain two alkyls on the $\alpha$-carbon of the hydroxyl group were prepared and used to give metal complexes such as $\mathrm{M}(\mathrm{mmp})_{4}(\mathrm{mmp}=1$-methoxy-2-methyl-2-propanolate; $\mathrm{M}=\mathrm{Zr}, \mathrm{Hf}),{ }^{11} \mathrm{M}(\mathrm{mmp})_{3}(\mathrm{M}=\mathrm{Cr}, \mathrm{Bi}),{ }^{9} \mathrm{M}(\mathrm{dmamp})_{3}(\mathrm{dmamp}$ = 1-dimethylamino-2-methyl-2-propanolate; $\mathrm{M}=\mathrm{Y}, \mathrm{Lu}){ }^{10}$ $\mathrm{Cu}(\mathrm{dmamp})_{2}{ }^{12}$ The ligands in the complexes have pendant groups capable of coordinating to metals as bidentate ligands. Some of trifunctional alcohols have been used for the preparation and homoleptic complexes of alkaline-earth elements ${ }^{13}$ as well as transition metals. ${ }^{14,15}$ For larger cations such as $\mathrm{Ba}^{2+}$ and $\mathrm{Sr}^{2+}$, it is necessary to introduce a neutral ancillary ligand possessing a sufficient number of donor sites or to incorporate a donor-functionalized feature into the anionic ligand to satisfy the multiple coordination requirement. Chi reported the successful preparation of alkalineearth metal complexes using a multi-donor site monoalcohol. ${ }^{16}$
Herein we disclose the synthesis and characterization of novel alcohols, which have pendant groups to stabilize the metal compounds by coordinating to the metal center in the formation of metal complexes. New alkoxyalcohols were prepared by the nucleophilic addition ring opening reaction between alkoxide anions and a highly reactive alkene oxide (epoxide).

\section{Results and Discussion}

1,2-Epoxy-2-methylpropane (isobutylene oxide) was introduced to afford the tertiary alcohols which have two methyl groups on the a-carbon of the oxygen atom of the alcohol. There have been many studies for usefulness of isobutylene oxide to make tertiary alcohols containing appropriate functional groups by reactions with carbanions such as alkyl, ${ }^{17-19}$ allyl, ${ }^{20,21}$ organocopper, ${ }^{22}$ fluorovinyl anion, ${ }^{23}$ acetylenide, ${ }^{24}$ and phosphonium ylide. ${ }^{25,26}$ Also, the reactions between isobutylene oxide and other nucleophiles such as alkoxide, ${ }^{27}$ thiolate, ${ }^{28}$ and phosphide anion ${ }^{26}$ have been applied to afford alkoxy alcohol, alkyl sulfide, and alkyl phosphine derivatives, respectively. Two methyl groups of tertiary alcohols might allow the increased volatility of metal compounds by causing steric hindrance to the oxygen atom, and thus preventing the neighboring alkoxide oxygen from approaching the metal center when the alkoxides form metal complexes.

The synthetic scheme for the donor-functionalized alkoxy alcohols is shown in Scheme 1. The typical reaction is that of 1,2-epoxy-2-methylpropane with the sodium alkoxide, which was prepared by the reaction of the corresponding alcohol with sodium or sodium hydride in THF. To complete the reaction, the reaction mixture was heated at the refluxing temperature. All compounds were easily prepared after work-up with water, by extraction with diethyl ether, followed by vacuum distillation. As an example of Scheme 1 , we carried out the synthesis of dmaempH. The treatment of a reactant alcohol, 2-(dimethylamino)ethanol with one equivalent of sodium hydride in THF leads to a solution of the corresponding sodium salt of the alkoxide anion. Addition of isobutylene oxide to the solution followed by refluxing the reaction mixture produced the derivative of 2methyl-2-propanolate anion containing the dimethylamino 


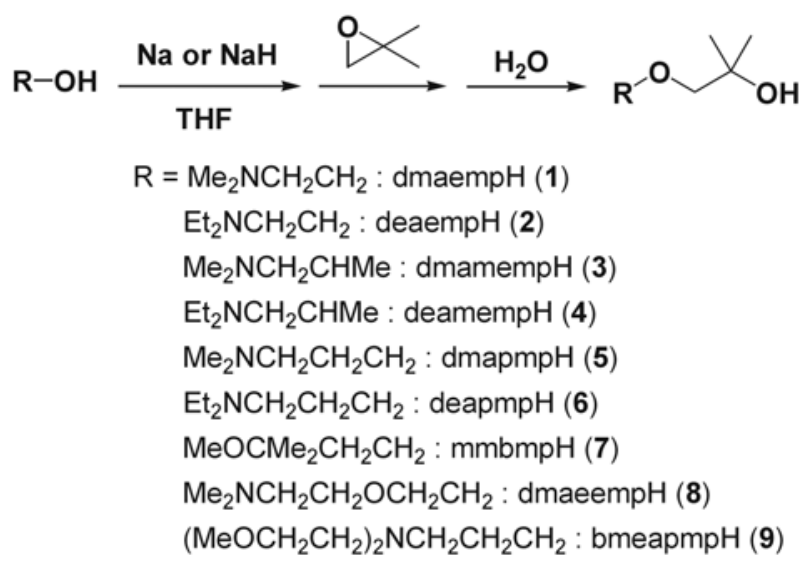

Scheme 1. Synthetic scheme of new donor-functionalized alcohols.

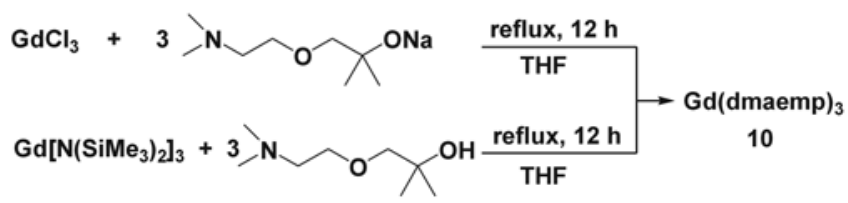

Scheme 2. Synthetic scheme of the Gd(dmaemp) $)_{3}$ complex.

group and ether linkage. Aqueous work-up, extraction, and vacuum distillation afforded a colorless liquid in moderate yield.

To investigate the potential usefulness of the new donorfunctionalized alcohols as ligands for metal complexes, we conducted the synthesis of a new metal complex, which contains one of the lanthanide metals and three equivalents of the alkoxide synthesized in this work. Refluxing the reaction mixture of gadolinium chloride and three equivalents of the sodium salt of dmaempH in THF gave a new viscous brown liquid gadolinium compound (Scheme 2). Another synthetic method for the gadolinium complex included the reaction between tris(bistrimethylsilylamido) gadolinium and 3 equivalents of dmaempH. Disappearance of the hydroxyl group frequency $\left(3398 \mathrm{~cm}^{-1}\right)$ in the IR spectrum of the prepared gadolinium complex confirmed the successful preparation of the compound. Also elemental analysis of the product is in good agreement with our suggested structure.

\section{Conclusions}

In summary, we designed and synthesized several donorfunctionalized alcohols by the reaction of isobutylene oxide and appropriate sodium salts of the alcohols. And a new gadolinium complex containing 3 dmaemp ligands has been successfully prepared for its application to gadolinium containing materials. The new donor-functionalized alcohols might be potentially useful ligands for the preparation of metal complexes as the precursors for materials. Other metal complexes and some application by MOCVD are under investigation in our group.

\section{Experimental Section}

All reactions were carried out under an inert atmosphere using standard Schlenk techniques. THF was distilled from sodium-benzophenone ketyl under nitrogen. Starting materials were purchased from Aldrich and TCI and used without further purification. The starting compound, $\left(\mathrm{MeOCH}_{2} \mathrm{CH}_{2}\right)_{2} \mathrm{NCH}_{2} \mathrm{CH}_{2} \mathrm{CH}_{2} \mathrm{OH}$ was prepared by $\mathrm{HCl}$ elimination reaction between bis(2-methoxyethyl)amine and 3-chloropropanol as described in the literature. ${ }^{29}$ NMR spectra were recorded on a Bruker DPX 300 spectrometer with deuterated solvents as an internal lock. ${ }^{1} \mathrm{H}(300 \mathrm{MHz})$ and ${ }^{13} \mathrm{C}(75 \mathrm{MHz})$ shifts are referenced to internal solvent resonances and reported relative to TMS. IR spectra were measured with a Nicolet NEXUS FT-IR spectrometer. Elemental analyses were conducted with Thermoquest EA$1110 \mathrm{CHN}$ analyzer in KRICT.

1-[(Dimethylamino)ethoxy]-2-methyl-2-propanol (dmaempH: 1). To a flame-dried Schlenk flask were placed sodium $(1.15 \mathrm{~g}, 50.0 \mathrm{mmol})$ and THF $(30 \mathrm{~mL})$. The flask was cooled to $0{ }^{\circ} \mathrm{C}$ and 2-(dimethylamino)ethanol $(5.03 \mathrm{~mL}$, $50.0 \mathrm{mmol})$ was slowly added. The reaction mixture was allowed to warm to room temperature while being stirred for $2 \mathrm{~h}$. To complete the reaction the reaction mixture was refluxed for an additional $5 \mathrm{~h}$. After cooling to $0{ }^{\circ} \mathrm{C}$, 2methyl-1-propenoxide (isobutylene oxide, $4.50 \mathrm{~mL}, 50.0$ $\mathrm{mmol})$ was slowly added. The reaction mixture was refluxed for $16 \mathrm{~h}$. After cooling to room temperature, water $(30 \mathrm{~mL})$ was added and the reaction mixture was stirred for $1 \mathrm{~h}$. A $\mathrm{NaCl}$-saturated aqueous solution $(20 \mathrm{~mL})$ and ether $(20 \mathrm{~mL})$ were added to the reaction mixture. The organic layer was separated, the aqueous layer was extracted with ether $(3 \times 20$ $\mathrm{mL})$, and the organic fractions were combined and dried over anhydrous magnesium sulfate. The solvent was evaporated to give a crude product. Purification was conducted by vacuum distillation to give a colorless liquid product in $52.0 \%$ yield $(4.19 \mathrm{~g}) .{ }^{1} \mathrm{H}$ NMR $\left(\mathrm{CDCl}_{3}\right): \delta=1.24(\mathrm{~s}, 6 \mathrm{H}$, $\left.\mathrm{C}\left(\mathrm{CH}_{3}\right)_{2}\right), 2.03\left(\mathrm{~s}, 6 \mathrm{H}, \mathrm{CH}_{3} \mathrm{~N}\right), 2.23(\mathrm{t}, J=5.51 \mathrm{~Hz}, 2 \mathrm{H}$, $\mathrm{NCH}_{2} \mathrm{CH}_{2}$ ), 3.17 (s, 2H, OCH $\left.\mathrm{O}_{2} \mathrm{C}\right), 3.37$ (t, $J=5.50 \mathrm{~Hz}, 2 \mathrm{H}$, $\left.\mathrm{CH}_{2} \mathrm{CH}_{2} \mathrm{O}\right), 4.33$ (bs, $\left.1 \mathrm{H}, \mathrm{OH}\right) .{ }^{13} \mathrm{C} \mathrm{NMR}\left(\mathrm{CDCl}_{3}\right): \delta=26.6$, 45.4, 59.1, 69.7, 69.8, 80.4. Anal. Calcd for $\mathrm{C}_{8} \mathrm{H}_{19} \mathrm{NO}_{2}$ : C, 59.59; H, 11.88; N, 8.69. Found: C, 59.03; H, 12.06; N, 9.47. FT-IR $\left(\mathrm{OH}, \mathrm{cm}^{-1}\right): v=3398$.

1-[(Diethylamino)ethoxy]-2-methyl-2-propanol (deaempH: 2). The same procedure as described for the preparation of compound 1 was followed using diethylaminoethanol instead of dimethylaminoethanol to give 2 in $66 \%$ yield. ${ }^{1} \mathrm{H}$ $\operatorname{NMR}\left(\mathrm{CDCl}_{3}\right): \delta=1.03\left(\mathrm{t}, J=7.5 \mathrm{~Hz}, 6 \mathrm{H}, \mathrm{CH}_{3} \mathrm{CH}_{2} \mathrm{~N}\right), 1.05$ $\left(\mathrm{s}, 6 \mathrm{H}, \mathrm{C}\left(\mathrm{CH}_{3}\right)_{2}\right), 2.59$ (q, $\left.J=6.9 \mathrm{~Hz}, 4 \mathrm{H}, \mathrm{CH}_{3} \mathrm{CH}_{2} \mathrm{~N}\right), 2.62$ $\left(\mathrm{t}, J=5.7 \mathrm{~Hz}, 2 \mathrm{H}, \mathrm{NCH}_{2} \mathrm{CH}_{2}\right), 3.33\left(\mathrm{~s}, 2 \mathrm{H}, \mathrm{OCH}_{2} \mathrm{C}\right), 3.64$ (t, $\left.J=5.7 \mathrm{~Hz}, 2 \mathrm{H}, \mathrm{CH}_{2} \mathrm{CH}_{2} \mathrm{O}\right) .{ }^{13} \mathrm{C} \mathrm{NMR}\left(\mathrm{CDCl}_{3}\right): \delta=11.4$, 26.3, 47.2, 53.4, 70.6, 71.0, 80.7. Anal. Calcd for $\mathrm{C}_{10} \mathrm{H}_{23} \mathrm{NO}_{2}$ : C, 63.45; H, 12.25; N, 7.40. Found: C, 62.31; H, $12.78 ; \mathrm{N}, 8.41$. FT-IR $\left(\mathrm{OH}, \mathrm{cm}^{-1}\right): v=3407$.

1-[2-(Dimethylamino)-1-methyl-ethoxy]-2-methyl-2-propanol (dmamempH: 3). The same procedure as described 
for the preparation of compound 1 was followed using 2(dimethylamino)-1-methyl-ethanol instead of dimethylaminoethanol to give 3 in $48.2 \%$ yield. ${ }^{1} \mathrm{H} \mathrm{NMR}\left(\mathrm{CDCl}_{3}\right): \delta$ $=1.04\left(\mathrm{~s}, 3 / 2 \mathrm{H}, \mathrm{NCH}_{2}\left(\mathrm{CH}_{3}\right)_{2} \mathrm{C}\right), 1.05\left(\mathrm{~s}, 3 \mathrm{H}, \mathrm{CH}_{2}\left(\mathrm{CH}_{3}\right)_{2} \mathrm{CO}\right)$, 1.06 (s, 3/2H, $\left.\mathrm{NCH}_{2}\left(\mathrm{CH}_{3}\right)_{2} \mathrm{C}\right), 1.16\left(\mathrm{~s}, 3 \mathrm{H}, \mathrm{CH}_{2}\left(\mathrm{CH}_{3}\right)_{2} \mathrm{CO}\right)$, $2.06\left(\mathrm{~d}, \mathrm{~J}=2.4 \mathrm{~Hz}, 1 / 2 \mathrm{H}, \mathrm{NCH}_{2}\left(\mathrm{CH}_{3}\right)\right), 2.11(\mathrm{~d}, J=2.4 \mathrm{~Hz}$, 1/2H, $\left.\mathrm{NCH}_{2}\left(\mathrm{CH}_{3}\right)\right), 2.24$ (s, 6H, $\left.\left(\mathrm{CH}_{3}\right)_{2} \mathrm{NCH}_{2}\right), 2.43$ (s, 1/ $\left.4 \mathrm{H}, \mathrm{NCH}_{2}\left(\mathrm{CH}_{3}\right)\right), 2.47\left(\mathrm{~d}, J=3.3 \mathrm{~Hz}, 1 / 2 \mathrm{H}, \mathrm{NCH}_{2}\left(\mathrm{CH}_{3}\right)\right)$, 2.51 (s, 1/4H, $\left.\mathrm{NCH}_{2}\left(\mathrm{CH}_{3}\right)\right), 3.14\left(\mathrm{~s}, 1 / 2 \mathrm{H}, \mathrm{OCH}_{2}\left(\mathrm{CH}_{3}\right)_{2} \mathrm{C}\right)$, $3.18\left(\mathrm{~s}, 1 / 2 \mathrm{H}, \mathrm{OCH}_{2}\left(\mathrm{CH}_{3}\right)_{2} \mathrm{C}\right), 3.50\left(\mathrm{~s}, 1 / 2 \mathrm{H}, \mathrm{OCH}_{2}\left(\mathrm{CH}_{3}\right)_{2} \mathrm{C}\right)$, $3.53\left(\mathrm{~s}, 1 / 2 \mathrm{H}, \mathrm{OCH}_{2}\left(\mathrm{CH}_{3}\right)_{2} \mathrm{C}\right), 3.61\left(\mathrm{~m}, 1 \mathrm{H},\left(\mathrm{CH}_{3}\right) \mathrm{CHOCH}_{2}\right)$, $6.22(\mathrm{bs}, 1 \mathrm{H}, \mathrm{OH}) .{ }^{13} \mathrm{C} \mathrm{NMR}\left(\mathrm{CDCl}_{3}\right): \delta=19.1,25.5,26.5$, 45.9, 66.3. 70.3, 76.5, 79.2. Anal. Calcd for $\mathrm{C}_{9} \mathrm{H}_{21} \mathrm{NO}_{2}$ : C, 61.67; H, 12.08; N, 7.99. Found: C, 61.13; H, 12.30; N, 8.99. FT-IR $\left(\mathrm{OH}, \mathrm{cm}^{-1}\right): v=3390$.

1-[2-(Diethylamino)-1-methyl-ethoxy]-2-methyl-2-propanol (deamempH: 4). The same procedure as described for the preparation of compound 1 was followed using 2(diethylamino)-1-methyl-ethanol instead of dimethylaminoethanol to give 4 in $43.5 \%$ yield. ${ }^{1} \mathrm{H} \mathrm{NMR}\left(\mathrm{CDCl}_{3}\right): \delta=1.00$ $\left(\mathrm{t}, J=6.0 \mathrm{~Hz}, 6 \mathrm{H},\left(\mathrm{C} \mathrm{H}_{3} \mathrm{CH}_{2}\right)_{2} \mathrm{~N}\right), 1.13(\mathrm{~d}, J=3.0 \mathrm{~Hz}, 3 \mathrm{H}$, $\left.\left(\mathrm{CH}_{3}\right) \mathrm{O}\right), 1.28\left(\mathrm{~s}, 6 \mathrm{H},\left(\mathrm{CH}_{3}\right)_{2} \mathrm{OH}\right), 2.32\left(\mathrm{~s}, 1 \mathrm{H}, \mathrm{NCH}_{2}\right), 2.40$ $\left(\mathrm{s}, 1 \mathrm{H}, \mathrm{NCH}_{2}\right), 2.56\left(\mathrm{q}, J=2.0 \mathrm{~Hz}, 2 \mathrm{H},\left(\mathrm{CH}_{3} \mathrm{CH}_{2}\right)_{2} \mathrm{~N}\right), 2.58$ $\left(\mathrm{q}, J=2 \mathrm{~Hz}, 2 \mathrm{H},\left(\mathrm{CH}_{3} \mathrm{CH}_{2}\right)_{2} \mathrm{~N}\right), 3.20\left(\mathrm{~s}, 1 \mathrm{H}, \mathrm{OCH}_{2}\right), 3.22$ (s, $\left.1 \mathrm{H}, \mathrm{OCH}_{2}\right), 3.67\left(\mathrm{~m}, J=1.5 \mathrm{~Hz}, 1 \mathrm{H}, \mathrm{NCH}_{2} \mathrm{CH}\right) .{ }^{13} \mathrm{C} \mathrm{NMR}$ $\left(\mathrm{CDCl}_{3}\right): \delta=11.0,19.0,25.3,26.6,47.4,60.9,70.6,78.1$, 79.5. Anal. Calcd for $\mathrm{C}_{11} \mathrm{H}_{25} \mathrm{NO}_{2}$ : C, 64.98; H, 12.39; N, 6.89. Found: C, 64.34; H, 12.43; N, 7.43. FT-IR $\left(\mathrm{OH}, \mathrm{cm}^{-1}\right)$ : $v=3401$.

1-[(3-Dimethylamino)propoxy]-2-methyl-2-propanol (dmapmpH: 5). The same procedure as described for the preparation of compound 1 was followed using 3dimethylaminopropanol instead of dimethylaminoethanol to give 5 in $44.3 \%$ yield. ${ }^{1} \mathrm{H}$ NMR $\left(\mathrm{CDCl}_{3}\right): \delta=1.49(\mathrm{~s}, 6 \mathrm{H}$, $\left.\mathrm{C}\left(\mathrm{CH}_{3}\right)_{2}\right), 1.71\left(\mathrm{~m}, J=6.3 \mathrm{~Hz}, 2 \mathrm{H}, \mathrm{CH}_{2} \mathrm{CH}_{2} \mathrm{CH}_{2}\right), 2.19$ (s, $\left.6 \mathrm{H}, \mathrm{CH}_{3} \mathrm{~N}\right), 2.38\left(\mathrm{t}, J=6.6 \mathrm{~Hz}, 2 \mathrm{H}, \mathrm{NCH}_{2}\right), 3.25(\mathrm{~s}, 2 \mathrm{H}$, $\left.\mathrm{OCH}_{2} \mathrm{C}\right), 3.61\left(\mathrm{t}, J=5.9 \mathrm{~Hz}, 2 \mathrm{H}, \mathrm{CH}_{2} \mathrm{CH}_{2} \mathrm{O}\right), 4.55(\mathrm{bs}, 1 \mathrm{H}$, $\mathrm{OH}) .{ }^{13} \mathrm{C} \mathrm{NMR}\left(\mathrm{CDCl}_{3}\right): \delta 26.1,27.4,45.3,56.4,70.2,70.4$, 79.3. Anal. Calcd for $\mathrm{C}_{9} \mathrm{H}_{21} \mathrm{NO}_{2}$ : C, 61.67; H, 12.08; N, 7.99. Found: C, 61.23; H, 12.24; N, 8.97. FT-IR $\left(\mathrm{OH}, \mathrm{cm}^{-1}\right)$ : $v=3417$.

1-[(3-Diethylamino)propoxy]-2-methyl-2-propanol (deapmpH: 6). The same procedure as described for the preparation of compound 1 was followed using 3-diethylaminopropanol instead of dimethylaminoethanol to give 6 in $52.9 \%$ yield. ${ }^{1} \mathrm{H}$ NMR $\left(\mathrm{CDCl}_{3}\right): \delta=0.99(\mathrm{t}, J=7.2 \mathrm{~Hz}, 6 \mathrm{H}$, $\left.\mathrm{CH}_{3} \mathrm{CH}_{2} \mathrm{~N}\right), 1.16$ (s. $\left.6 \mathrm{H}, \mathrm{C}\left(\mathrm{CH}_{3}\right)_{2}\right), 1.71$ (t, $J=6.5 \mathrm{~Hz}, 2 \mathrm{H}$, $\left.\mathrm{CH}_{2} \mathrm{CH}_{2} \mathrm{CH}_{2}\right), 2.49\left(\mathrm{~m}, 6 \mathrm{H}, \mathrm{CH}_{2} \mathrm{NCH}_{2}\right), 3.23(\mathrm{~s}, 2 \mathrm{H}$, $\left.\mathrm{OCH}_{2} \mathrm{C}\right), 3.55$ (t, $\left.\mathrm{J}=6.0 \mathrm{~Hz}, 2 \mathrm{H}, \mathrm{CH}_{2} \mathrm{CH}_{2} \mathrm{O}\right) .{ }^{13} \mathrm{C} \mathrm{NMR}$ $\left(\mathrm{CDCl}_{3}\right): \delta=11.2,26.2,27.1,46.4,49.5,70.2,70.3,79.2$. Anal. Calcd for $\mathrm{C}_{11} \mathrm{H}_{25} \mathrm{NO}_{2}$ : C, 64.98; H, 12.39; N, 6.89 . Found: C, 64.96; H, 12.45; N, 8.01. FT-IR $\left(\mathrm{OH}, \mathrm{cm}^{-1}\right): v=$ 3425 .

3-(3-Methoxy-3-methyl-butoxy)-2-methyl-2-propanol (mmbmpH: 7). The same procedure as described for the preparation of compound 1 was followed using 3-methoxy- 3-methyl-butanol instead of dimethylaminoethanol to give 7 in $56.8 \%$ yield. ${ }^{1} \mathrm{H}$ NMR $\left(\mathrm{CDCl}_{3}\right): \delta=1.13(\mathrm{~s}, 6 \mathrm{H}$, $\left.\mathrm{C}\left(\mathrm{CH}_{3}\right)_{2} \mathrm{OH}\right), 1.14\left(\mathrm{~s}, 6 \mathrm{H}, \mathrm{CH}_{3} \mathrm{OC}\left(\mathrm{CH}_{3}\right)_{2}\right), 1.75(\mathrm{t}, J=6.9$ $\left.\mathrm{Hz}, 2 \mathrm{H}, \mathrm{CCH}_{2} \mathrm{CH}_{2} \mathrm{O}\right), 2.51$ (bs, $\left.1 \mathrm{H}, \mathrm{OH}\right), 3.14$ (s, 3H, $\left.\mathrm{CH}_{3} \mathrm{O}\right), 3.19\left(\mathrm{~s}, 2 \mathrm{H}, \mathrm{OCH}_{2} \mathrm{C}\left(\mathrm{CH}_{3}\right)_{2}\right), 3.52(\mathrm{t}, \mathrm{J}=6.9 \mathrm{~Hz}, 2 \mathrm{H}$, $\left.\mathrm{CH}_{2} \mathrm{CH}_{2} \mathrm{O}\right) .{ }^{13} \mathrm{C} \mathrm{NMR}\left(\mathrm{CDCl}_{3}\right): \delta=25.4,26.2,39.2,49.2$, 67.9, 70.1, 73.9, 79.3. Anal. Calcd for $\mathrm{C}_{11} \mathrm{H}_{24} \mathrm{O}_{3}$ : C, 64.67; H, 11.84. Found: C, 62.50; H, 11.93. FT-IR $\left(\mathrm{OH}, \mathrm{cm}^{-1}\right): v=$ 3446.

1-[2-[2-(Dimethylamino)ethoxy]ethoxy]-2-methyl-2-propanol (dmaeempH: 8). The same procedure as described for the preparation of compound 1 was followed using 2-[2(dimethylamino)ethoxy]ethanol instead of dimethylaminoethanol to give 8 in $75.6 \%$ yield. ${ }^{1} \mathrm{H} \mathrm{NMR}\left(\mathrm{CDCl}_{3}\right): \delta=1.16$ (s, 6H, C(CH3) $)_{2}, 2.23\left(\mathrm{~s}, 6 \mathrm{H}, \mathrm{NCH}_{3}\right), 2.47$ (t, $J=5.8 \mathrm{~Hz}$, $\left.2 \mathrm{H}, \mathrm{NCH}_{2}\right), 2.87$ (bs, $\left.1 \mathrm{H}, \mathrm{OH}\right), 3.29\left(\mathrm{~s}, 2 \mathrm{H}, \mathrm{OCH}_{2} \mathrm{C}\left(\mathrm{CH}_{3}\right)_{2}\right)$, $3.56\left(\mathrm{~m}, 6 \mathrm{H}, \mathrm{CH}_{2} \mathrm{OCH}_{2} \mathrm{CH}_{2} \mathrm{O}\right) .{ }^{13} \mathrm{C} \mathrm{NMR}\left(\mathrm{CDCl}_{3}\right): \delta=26.1$, 46.0, 58.9, 69.5, 70.2, 70.4, 71.2, 79.8. Anal. Calcd for $\mathrm{C}_{10} \mathrm{H}_{23} \mathrm{NO}_{3}$ : C, 58.50; H, 11.29; N, 6.82. Found: C, 58.42; H, 11.47; N, 7.84. FT-IR $\left(\mathrm{OH}, \mathrm{cm}^{-1}\right): v=3419$.

1-[3-Bis(2-methoxyethylamino)propoxy]-2-methyl-2propanol (bmeapmpH: 9). The same procedure as described for the preparation of compound 1 was followed using 3-bis(2-methoxyethylamino)propanol instead of dimethylaminoethanol to give 9 in $43.5 \%$ yield. ${ }^{1} \mathrm{H}$ NMR $\left(\mathrm{CDCl}_{3}\right): \delta=1.17\left(\mathrm{~s}, 6 \mathrm{H}, \mathrm{C}\left(\mathrm{CH}_{3}\right)_{2}\right), 1.72$ (quintet, $J=6.75$ $\left.\mathrm{Hz}, 2 \mathrm{H}, \mathrm{CH}_{2} \mathrm{CH}_{2} \mathrm{CH}_{2}\right), 2.62(\mathrm{t}, J=7.35 \mathrm{~Hz}, 2 \mathrm{H}$, $\left.\mathrm{NCH}_{2} \mathrm{CH}_{2} \mathrm{CH}_{2} \mathrm{O}\right), 2.69\left(\mathrm{t}, J=6.0 \mathrm{~Hz}, 4 \mathrm{H}, \mathrm{OCH}_{2} \mathrm{CH}_{2} \mathrm{~N}\right)$, $3.22\left(\mathrm{~s}, 2 \mathrm{H}, \mathrm{OCH}_{2} \mathrm{C}\left(\mathrm{CH}_{3}\right)_{2}\right), 3.32\left(\mathrm{~s}, 6 \mathrm{H}, \mathrm{OCH}_{3}\right), 3.44(\mathrm{t}, J=$ $\left.6.3,4 \mathrm{H}, \quad \mathrm{OCH}_{2} \mathrm{CH}_{2} \mathrm{~N}\right), 3.50(\mathrm{t}, J=6.0 \mathrm{~Hz}, 2 \mathrm{H}$, $\left.\mathrm{NCH}_{2} \mathrm{CH}_{2} \mathrm{CH}_{2} \mathrm{O}\right) .{ }^{13} \mathrm{C} \mathrm{NMR}\left(\mathrm{CDCl}_{3}\right): \delta=26.2,27.2,52.0$, 53.9, 58.9, 69.8. Anal. Calcd for $\mathrm{C}_{13} \mathrm{H}_{29} \mathrm{NO}_{4}$ : C, 59.28; H, 11.10; N, 5.32. Found: C, 58.69; H, 11.45; N, 6.22. FT-IR $\left(\mathrm{OH}, \mathrm{cm}^{-1}\right): v=3453$.

Gadolinium(III) tris[1-[2-(dimethylamino)ethoxy]-2methyl-2-propoxide] (Gd(dmaemp) $\left.)_{3}: 10\right)$. A flame-dried $100 \mathrm{~mL}$ Schlenk flask was charged with $\mathrm{GdCl}_{3}(0.48 \mathrm{~g}, 1.82$ $\mathrm{mmol})$ and THF $(50 \mathrm{~mL})$. To this solution was slowly added a solution of the sodium salt of dmaempH (1.00 g, 5.46 $\mathrm{mmol})$ in THF $(20 \mathrm{~mL})$ at room temperature. The reaction mixture became cloudy after stirring for $12 \mathrm{~h}$. The resultant was filtered off and the filtrate was evaporated under vacuum to dryness to yield a viscous brown liquid $(0.95 \mathrm{~g}$, $82.0 \%$ ). Another method for the synthesis of the compound 10 was the reaction of tris(bistrimethylsilylamido) gadolinium with 3 equivalents of dmaempH in THF at the refluxing temperature with similar yield. Anal. Calcd for $\mathrm{C}_{24} \mathrm{H}_{54} \mathrm{~N}_{3} \mathrm{O}_{6} \mathrm{Gd}$ : C, 45.18; H, 8.53; N, 6.59. Found: C, 44.72; H, 8.66; N, 6.59. FT-IR $\left(\mathrm{cm}^{-1}\right): v=2964,2862,2819,2769$, $1460,1182,1114$.

Acknowledgments. We thank the Ministry of Science and Technology of Korea for financial support through the National Research Laboratory (NRL) Program and the Programs of NT-IT Fusion Strategy of Advanced Technologies. 


\section{References}

1. Hubert-Pfalzgraf, L. G. Coord. Chem. Rev. 1998, 178-180, 967.

2. Spencer, J. T. In Progress in Inorganic Chemistry, Vol. 41; Karlin, K. D., Ed.; John Wiley \& Sons, Inc: 1994.

3. Bae, B.-J.; Lee, K.; Seo, W. S.; Miah, Md. A.; Kim, K.-C.; Park, J. T. Bull. Korean Chem. Soc. 2004, 25, 1661.

4. Lim, J. T.; Hong, S. T.; Lee, J. C.; Lee, I.-M. Bull. Korean Chem. Soc. 1996, 17, 1023.

5. Hong, S. T.; Lim, J. T.; Lee, J. C.; Xue, M.; Lee, I.-M. Bull. Korean Chem. Soc. 1996, 17, 637.

6. Wojtczak, W. A.; Fleig, P. F.; Hampden-Smith, M. J. $A d v$. Organomet. Chem. 1996, 40, 215.

7. Sung, M. M.; Kim, C. G.; Kim, Y. Bull. Korean Chem. Soc. 2004, 25,480 .

8. Herrmann, W. A.; Anwander, R.; Denk, M. Chem. Ber. 1992, 125, 2399.

9. Herrmann, W. A.; Huber, N. W.; Anwander, R.; Priermeier, T. Chem. Ber. 1993, 126, 1127.

10. Anwander, R.; Munck, F. C.; Priermeier, T.; Scherer, W.; Runte, O.; Herrmann, W. A. Inorg. Chem. 1997, 36, 3545.

11. Williams, P. A.; Roberts, J. L.; Jones, A. C.; Chalker, P. R.; Tobin, N. L.; Bickley, J. F.; Davies, H. O.; Smith, L. M.; Leedham, T. J. Chem. Vap. Deposition 2002, 8, 163.

12. Park, J. W.; Jang, H. S.; Kim, M.; Sung, K.; Lee, S. S.; Chung, T.M.; Koo, S.; Kim, C. G.; Kim, Y. Inorg. Chem. Commun. 2004, 7, 463.

13. Herrmann, W. A.; Huber, N. W.; Priermeier, T. Angew. Chem. Int.
Ed. Engl. 1994, 33, 105.

14. Chi, Y.; Hsu, P.-F.; Liu, C.-S.; Ching, W.-L.; Chou, T.-Y.; Carty, A. J.; Peng, S.-M.; Lee, G.-H.; Chuang, S.-H. J. Mater. Chem. 2002, 12,3541 .

15. Herrmann, W. A.; Huber, N. W. Chem. Ber. 1994, 127, 821.

16. Chi, Y.; Ranjan, S.; Chou, T.-Y.; Liu, C.-S.; Peng, S.-M.; Lee, G.H. J. Chem. Soc., Dalton Trans. 2001, 2462.

17. Murai, T.; Aso, H.; Kato, S. Org. Lett. 2002, 4, 1407.

18. Ahlbrecht, H.; Kornetzky, D. Synthesis 1988, 775.

19. Imai, T.; Nishida, S.; Tsuji, T. J. Chem. Soc., Chem. Commun. 1994, 2353.

20. Akiyama, T.; Ishida, Y. Synlett 1998, 1150.

21. Ongoka, P.; Mauzé, B.; Miginiac, L. J. Organomet. Chem. 1985 284, 139.

22. Alexakis, A.; Jachiet, D.; Normant, J. F. Tetrahedron 1986, 42, 5607.

23. Dubuffet, T.; Sauvêtre, R.; Normant, J.-F. J. Organomet. Chem. 1988, 341, 11 .

24. Mandai, T.; Matsumoto, T.; Kawada, M.; Tsuji, J. Tetrahedron 1994, 50, 475 .

25. Andrews, D. R.; Barton, D. H. R.; Hesse, R. H.; Pechet, M. M. J. Org. Chem. 1986, 51, 4819 .

26. Baxter, S. M.; Wolczanski, P. T. Organometallics 1990, 9, 2498.

27. Ikeda, I.; Yamamura, S.; Nakatsuji, Y.; Okahara, M. J. Org. Chem. 1980, 45, 5355.

28. Malmström, J.; Gupta, V.; Engman, L. J. Org. Chem. 1998, 63, 3318.

29. Tilford, C. H.; Shelton, R. S.; Van Campen, M. G. Jr. J. Am. Chem. Soc. 1948, 70, 4001. 